pathogens describing our current understanding of disease burden and the potential for vaccines as potential interventions. Many countries in Africa have documented the high burden of rotavirus. Almost 30 countries have introduced rotavirus vaccines with or without Gavi support, and recent studies document dramatic reductions in diarrhoeal hospitalizations and diarrhoeal deaths post introduction.

Norovirus is a ubiquitous virus causing diarrhoeal disease in all age ranges, although the incidence is highest in young children. Although a common cause of diarrhoea, norovirus is often associated with asymptomatic shedding making it difficult to ascertain the true burden of the disease. Nevertheless, advances towards understanding the epidemiology and diversity of norovirus in Africa are important to inform future vaccine efforts.

EAds have recently been described as one of the top 5 pathogens associated with acute severe diarrhoea in young children $<5$ years in Africa and Asia. Coupled with the high prevalence of EAds in HIV-infected children requires more focused research, although there is very limited vaccine development currently.

HEV is associated with water-borne and zoonotic infections, and is reported in large outbreaks. It has high mortality in pregnant women and is associated with high rates of stillbirths. Little is known of the extent of HEV infection in Africa. A Chinese vaccine against HEV has been licensed and WHO has identified gaps in research that are required for future immunization opportunities.

\section{OA-018 THE BURDEN AND FUTURE SCOPE FOR IMPORTANT VIRAL ENTERIC PATHOGENS IN AFRICA}

Duncan Steele. Bill \& Melinda Gates Foundation, United States of America

\subsection{6/bmjgh-2016-000260.25}

Background The burden of viral enteric infections in Africa is enormous, although poorly described. Some, such as rotavirus, are well recognized, whereas others including norovirus or enteric adenovirus (EAd), show high burden which is only now being described. Hepatitis E virus (HEV) is a neglected enteric viral infection although is on WHO's radar screen as an important pathogen which needs further evaluation in Africa and Asia. This presentation will focus on these four enteric viral 